\title{
Search for Weakly Interacting Massive Particles with the First Five-Tower Data from the Cryogenic Dark Matter Search at the Soudan Underground Laboratory
}

Z. Ahmed, ${ }^{2}$ D. S. Akerib, ${ }^{3}$ S. Arrenberg, ${ }^{16}$ M. J. Attisha, ${ }^{1}$ C. N. Bailey, ${ }^{3}$ L. Baudis, ${ }^{16}$ D. A. Bauer,${ }^{4}$ J. Beaty, ${ }^{15}$ P. L. Brink, ${ }^{9}$ T. Bruch, ${ }^{16}$ R. Bunker, ${ }^{12}$ S. Burke, ${ }^{12}$ B. Cabrera, ${ }^{9}$ D. O. Caldwell, ${ }^{12}$ J. Cooley,${ }^{9}$ P. Cushman,${ }^{15}$ F. DeJongh, ${ }^{4}$ M. R. Dragowsky, ${ }^{3}$ L. Duong,,${ }^{15}$ J. Emes,${ }^{5}$ E. Figueroa-Feliciano,${ }^{6}$ J. Filippini, ${ }^{11}$ M. Fritts, ${ }^{15}$ R. J. Gaitskell, ${ }^{1}$ S. R. Golwala, ${ }^{2}$ D. R. Grant, ${ }^{3}$ J. Hall, ${ }^{4}$ R. Hennings-Yeomans, ${ }^{3}$ S. Hertel, ${ }^{6}$ D. Holmgren, ${ }^{4}$ M. E. Huber, ${ }^{13}$ R. Mahapatra, ${ }^{12}$ V. Mandic, ${ }^{15}$ K. A. McCarthy, ${ }^{6}$ N. Mirabolfathi, ${ }^{11}$ H. Nelson, ${ }^{12}$ L. Novak, ${ }^{9}$ R. W. Ogburn, ${ }^{9}$ M. Pyle,,${ }^{9}$ X. Qiu, ${ }^{15}$ E. Ramberg, ${ }^{4}$ W. Rau, ${ }^{7}$ A. Reisetter ${ }^{15}$ T. Saab ${ }^{14}$ B. Sadoulet, ${ }^{5,11}$ J. Sander,${ }^{12}$ R. Schmitt, ${ }^{4}$ R. W. Schnee, ${ }^{10}$ D. N. Seitz, ${ }^{11}$ B. Serfass, ${ }^{11}$ A. Sirois, ${ }^{3}$ K. M. Sundqvist,${ }^{11}$ M. Tarka, ${ }^{16}$ A. Tomada,${ }^{9}$ G. Wang, ${ }^{2}$ S. Yellin, ${ }^{9,12}$ J. Yoo, ${ }^{4}$ and B. A. Young ${ }^{8}$

(CDMS Collaboration)

\author{
${ }^{1}$ Department of Physics, Brown University, Providence, Rhode Island 02912, USA \\ ${ }^{2}$ Department of Physics, California Institute of Technology, Pasadena, California 91125, USA \\ ${ }^{3}$ Department of Physics, Case Western Reserve University, Cleveland, Ohio 44106, USA \\ ${ }^{4}$ Fermi National Accelerator Laboratory, Batavia, Illinois 60510, USA \\ ${ }^{5}$ Lawrence Berkeley National Laboratory, Berkeley, California 94720, USA \\ ${ }^{6}$ Department of Physics, Massachusetts Institute of Technology, Cambridge, Massachusetts 02139, USA \\ ${ }^{7}$ Department of Physics, Queen's University, Kingston, ON, K7L 3N6, Canada \\ ${ }^{8}$ Department of Physics, Santa Clara University, Santa Clara, California 95053, USA \\ ${ }^{9}$ Department of Physics, Stanford University, Stanford, California 94305, USA \\ ${ }^{10}$ Department of Physics, Syracuse University, Syracuse, New York 13244, USA \\ ${ }^{11}$ Department of Physics, University of California, Berkeley, California 94720, USA \\ ${ }^{12}$ Department of Physics, University of California, Santa Barbara, California 93106, USA \\ ${ }^{13}$ Departments of Physics \& Elecectrical Engineering, University of Colorado Denver, Denver, Colorado 80217, USA \\ ${ }^{14}$ Department of Physics, University of Florida, Gainesville, Florida 32611, USA \\ ${ }^{15}$ School of Physics \& Astronomy, University of Minnesota, Minneapolis, Minnesota 55455, USA \\ ${ }^{16}$ Physics Institute, University of Zürich, Zürich, Switzerland \\ (Received 28 March 2008; published 5 January 2009)
}

\begin{abstract}
We report results from the Cryogenic Dark Matter Search at the Soudan Underground Laboratory (CDMS II) featuring the full complement of 30 detectors. A blind analysis of data taken between October 2006 and July 2007 sets an upper limit on the weakly interacting massive particle (WIMP) nucleon spinindependent cross section of $6.6 \times 10^{-44} \mathrm{~cm}^{2}\left(4.6 \times 10^{-44} \mathrm{~cm}^{2}\right.$ when combined with previous CDMS II data) at the $90 \%$ confidence level for a WIMP mass of $60 \mathrm{GeV} / c^{2}$. This achieves the best sensitivity for dark matter WIMPs with masses above $44 \mathrm{GeV} / c^{2}$, and significantly restricts the parameter space for some favored supersymmetric models.
\end{abstract}

DOI: 10.1103/PhysRevLett.102.011301

PACS numbers: 95.35.+d, 14.80.Ly

Cosmological observations [1] imply the existence of nonbaryonic dark matter that drives structure formation on large scales and dominates galactic and extra-galactic dynamics. Weakly Interacting Massive Particles (WIMPs) [2], with masses between a few tens of $\mathrm{GeV} / c^{2}$ and a few $\mathrm{TeV} / c^{2}$, form a generic class of dark matter candidates, motivated $[3,4]$ both by the measured value of the cosmological density and by the need to stabilize the standard model of particle physics at the weak scale.

WIMPs should be distributed in a halo surrounding the Milky Way and scatter in terrestrial particle detectors [5,6]. Their coherent scattering on nuclei should lead to a roughly exponential energy-transfer spectrum with a mean recoil energy in the tens of $\mathrm{keV}[4,7]$. The event rate is expected to be below 0.1 event per kilogram of target per day, much smaller than radioactivity rates in most materials. A number of technologies, most based on the identification of nuclear recoils among the electron recoils produced by $\gamma$-rays and betas from radioactivity, are starting to reach this sensitivity level, corresponding to a spin-independent WIMP-nucleon scattering cross section $\mathcal{O}\left(10^{-43}\right) \mathrm{cm}^{2}$. No dark matter signal has yet been observed except the recently reinforced claim by the DAMA/LIBRA collaboration [8], which cannot easily be reconciled with the WIMP hypothesis given the null results of all other direct WIMPsearch experiments. These experiments are complemented 
by searches for WIMP-annihilation products and searches for new physics (e.g., supersymmetry or extra dimensions) at particle colliders [9].

The Cryogenic Dark Matter Search at the Soudan Underground Laboratory (CDMS II) operates a total of $19 \mathrm{Ge}(250 \mathrm{~g}$ each) and $11 \mathrm{Si}(100 \mathrm{~g}$ each) solid-state detectors at $\sim 40 \mathrm{mK}[10,11]$. These detectors are disks, $7.6 \mathrm{~cm}$ in diameter and $1 \mathrm{~cm}$ thick, and arranged in five towers (vertical stacks of six detectors). Ionizing radiation produces electrons and holes together with phonons. The charge carriers are drifted by a small electric field $(3 \mathrm{~V} / \mathrm{cm})$ and collected on two concentric electrodes on one flat face. Phonons are collected before thermalizing using four superconducting thin-film circuits, each covering a quadrant on the other flat face. The ratio of ionization to phonon recoil energy ("ionization yield") allows us to discriminate nuclear from electron recoils with a rejection factor of $>10^{4}$ [11]. Electron recoils within $\sim 10 \mu \mathrm{m}$ of the detector surface suffer from suppressed ionization yield, sufficient to misclassify a surface electron recoil as a nuclear recoil. Signal timing provides effective discrimination against these events, improving our overall rejection of electron recoils to $>10^{6}$. To reduce the external $\gamma$-ray and neutron backgrounds, the experimental setup [11] includes $\mathrm{Pb}$ and polyethylene shielding, which is surrounded by a scintillator veto to reject events caused by cosmogenic muons or showers.

We report on data from two periods (Runs 123 and 124) between October 2006 and July 2007. Improvements made since our previous publications [12] include deployment of three additional towers containing 13 (5) Ge (Si) detectors, greater cryogenic stability, faster data acquisition, enhanced monitoring and control of data quality, and improved analysis techniques. Because of the considerably lower sensitivity of the $\mathrm{Si}$ detectors, we consider in this analysis only the Ge detectors for the WIMP search. Of the 19 Ge detectors, three suffered reduced performance from readout failures and one had poor energy resolution, and these have been left out of this report. The remaining $15 \mathrm{Ge}$ detectors $(3.75 \mathrm{~kg})$ were used for the Run 123 analysis. Run 124 followed a partial warm-up of the cryostat to $10 \mathrm{~K}$, causing eight of these detectors to have differences in performance between the two runs. In the interest of a timely dissemination of the results, the analysis of data taken with these eight detectors during the shorter Run 124 has been postponed.

Extensive calibrations with $\gamma$-ray $\left({ }^{133} \mathrm{Ba}\right)$ and neutron $\left({ }^{252} \mathrm{Cf}\right)$ sources were used to determine data selection criteria ("cuts") that define the signal region and to characterize detector performance and stability. Calibration data taken regularly with ${ }^{133} \mathrm{Ba}$ generated over $28 \times 10^{6}$ electron-recoil events between $10-100 \mathrm{keV}$, exceeding by a factor of 30 the number of comparable events in the WIMP-search data. Alternating events from the ${ }^{133} \mathrm{Ba}$ data were separated into two statistically independent samples to allow unbiased characterization of cut performance. Neutron calibration during five separate periods produced more than $10^{5}$ nuclear recoils used to characterize WIMP acceptance.

Both calibration and WIMP-search data were used to study detector stability and identify periods of poor performance. We discarded data sets with significant deviations from a standard set of one- and two-dimensional event parameter distributions. Periods of degraded ionization collection are of particular concern, as bulk electronrecoil events may leak into the signal region, as occurred in our most recently published analysis [12]. A cut excludes individual detectors from the WIMP search when the lowyield event fraction exceeds the mean (calculated over the full analysis period) by $2 \sigma$.

The phonon energy scale and the timing of the phonon signals vary slightly depending on the position and energy of each event. In order to maintain effective surface-event rejection, we compensate for these variations using an empirical lookup table based on our electron-recoil calibrations. The present analysis incorporates for the first time energy dependencies into this lookup table, enabling improved surface-event discrimination.

Event reconstruction at large radius remains imperfect due to degeneracies in the phonon position quantities. A small number of high-radius events suffered from miscalibration due to these degeneracies. A cut on the positionrelated phonon quantities removes events in problematic regions of the lookup table. We also remove events from the outer part of each detector by a fiducial volume cut based on the partitioning of energy between the two concentric charge electrodes.

For each event, we measure the rise time of the largest phonon pulse and the delay relative to the ionization signal. The sum of the rise time and delay provides good rejection of surface electron-recoil events while retaining reasonable acceptance of nuclear recoils. Figure 1 shows the distribution of this "timing parameter" in calibration data ( $\gamma$-rays, $\gamma$-ray-induced surface events and neutron-induced nuclear recoils) in the energy range $10-100 \mathrm{keV}$. To effectively remove surface events, we require that candidate dark matter events exceed a minimum value for the timing parameter ("timing cut"), determined for each detector by setting an allowed maximum passage fraction for surface events in a subset of the ${ }^{133} \mathrm{Ba}$ calibration data. We also require that WIMP candidates be consistent with the nuclear-recoil event distribution (i.e., the difference between delay and rise time is less than a $4 \sigma$ deviation from the neutron distribution mean). The performance of this cut is superior to that of earlier analyses due to improvements to the lookup table.

We require that a candidate dark matter event deposit significant energy ( $>4 \sigma$ above mean noise) in one and only one detector ("single scatter event"), since WIMPs will not interact more than once in our apparatus. All 30 


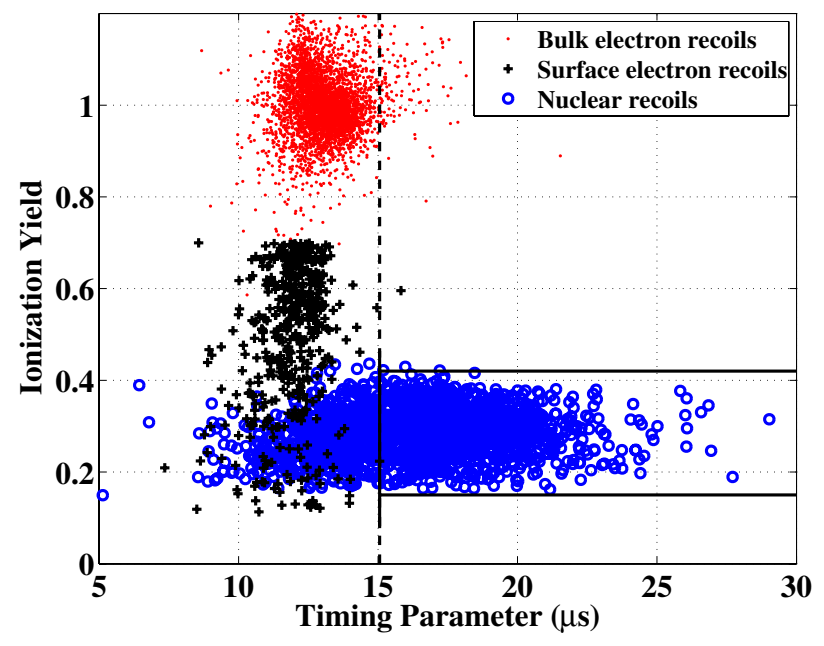

FIG. 1 (color online). Ionization yield versus timing parameter (see text) for calibration data in one of our Ge detectors. The yield is normalized to unity for typical bulk-electron recoils (dots; from ${ }^{133} \mathrm{Ba} \gamma$-rays). Low-yield ${ }^{133} \mathrm{Ba}$ events $(+)$, attributed to surface electron recoils, are discriminated from neutroninduced nuclear recoils from ${ }^{252} \mathrm{Cf}(\bigcirc)$, based on timing parameter values. The vertical dashed line indicates the minimum timing parameter allowed for candidate dark matter events in this detector, and the box shows the approximate signal region, which is in fact weakly energy dependent.

detectors contributed to active vetoing of multiple scatter events at all times. We further require that a WIMP candidate shows no significant activity in the surrounding scintillator veto shield during a $200-\mu$ s window around the trigger. Candidates must also lie within the $2 \sigma$ region of the nuclear-recoil distribution in ionization yield.

The acceptance of our analysis cuts for single-scatter nuclear recoils was measured as a function of energy based on both neutron calibration and WIMP-search data. Most cuts have very little effect on our acceptance of true nuclear recoils, with the ionization-based fiducial volume and phonon-timing cuts imposing the highest costs in signal acceptance, both measured on neutron calibration data. The acceptance is $\sim 31 \%$ and nearly constant above $15 \mathrm{keV}$ and falls to $\sim 24 \%$ at $10 \mathrm{keV}$ due to reduced trigger efficiency. The exposure of this analysis is $397.8 \mathrm{~kg}$ days before and $121.3 \mathrm{~kg}$ days after all cuts (averaged over recoil energies 10-100 keV, weighted for a WIMP mass of $60 \mathrm{GeV} / c^{2}$ ).

To avoid bias, we performed a blind analysis. An event mask was defined during initial data reduction to remove events in and near the signal region from WIMP-search data sets while developing the analysis. This mask was based on primary quantities not subject to refinement during the analysis process. After WIMP selection criteria were finalized, the masking was relaxed to cover only the actual signal region to aid in background estimation.

Surface events mainly occur due to radioactive contamination on detector surfaces or as a result of external $\gamma$-ray interactions releasing low-energy electrons from surfaces near the detectors. A correlation analysis between alphadecay and surface-event rates provides evidence that ${ }^{210} \mathrm{~Pb}$ (a daughter-product of ${ }^{222} \mathrm{Rn}$ ) is a major component of our surface-event background [13]. Surface events generated in situ at Soudan, either from calibration with a ${ }^{133} \mathrm{Ba}$ source or naturally present in the WIMP-search data, were studied to understand the surface-event leakage into the signal region. We estimate the surface-event leakage based upon the observed numbers of single- and multiplescatter events in each detector within and surrounding the $2 \sigma$ nuclear-recoil band. Our estimate treats "exterior" detector surfaces (those that do not face another detector) separately from "interior" surfaces, since interior surfaces have high sensitivity to low-energy multiplescatter events arising from particle interactions at the surfaces of adjacent detectors. The expected background due to surface interactions in this WIMP-search analysis is $0.6_{-0.3}^{+0.5}(\text { stat })_{-0.2}^{+0.3}$ (syst) event.

Neutrons induced by radioactive processes or by cosmic-ray muons interacting near the apparatus can generate nuclear-recoil events that cannot be distinguished from possible dark matter interactions on an event-byevent basis. Monte Carlo simulations of the cosmic-ray muons and subsequent neutron production and transport have been conducted with FLUKA [14], MCNPX [15], and GEANT4 [16] to estimate this cosmogenic neutron background. Normalizing the results to the observed vetocoincident multiple-scatter nuclear-recoil rate leads to a conservative upper limit on this background of $<0.1$ event in our WIMP-search data.

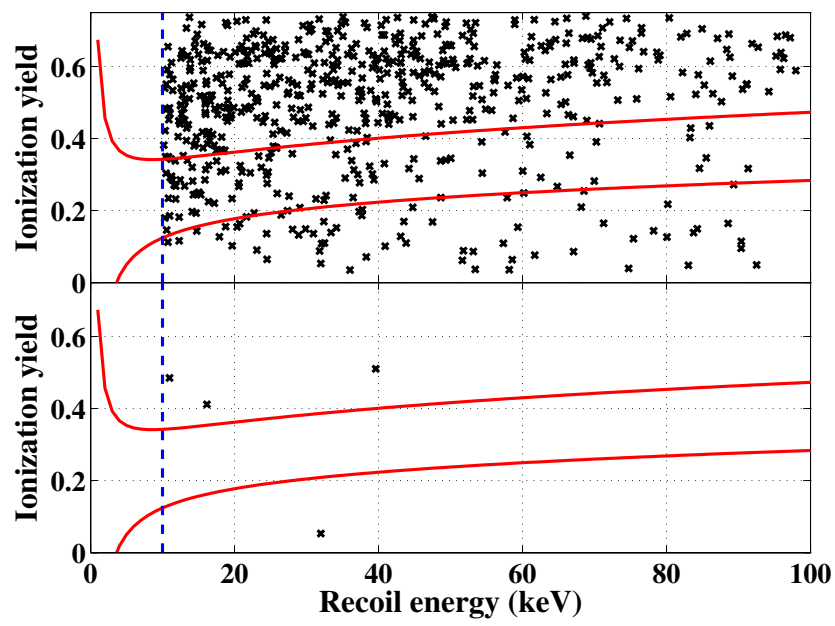

FIG. 2 (color online). Top: Ionization yield versus recoil energy in all detectors included in this analysis for events passing all cuts except the timing cut. The signal region between 10 and $100 \mathrm{keV}$ recoil energies was defined using neutron calibration data and is indicated by the curved lines. Bulk-electron recoils have yield near unity and are above the vertical scale limits. Bottom: Same, but after applying the timing cut. No events are found within the signal region. 
Monte Carlo simulations of neutrons induced by nuclear decay were based on $\gamma$-ray measurements of $U$ and $T h$ daughter products in the materials of our experimental setup and by assuming secular equilibrium. The estimated background is $<0.1$ event, dominated by the deduced upper limit of $\mathrm{U}$ in the $\mathrm{Pb}$ shield. Direct measurements of $\mathrm{U}$ in $\mathrm{Pb}$ [17] from the same source as the $\mathrm{Pb}$ used in our shield suggest a considerably lower contamination.

After all analysis cuts were finalized and leakage estimation schemes selected, we unmasked the WIMP-search signal region. No event was observed within this region. Figure 2 is a compilation of the low-yield events observed in all detectors used in this analysis. The upper panel shows the ionization yield distribution versus energy for singlescatter events passing all data selection cuts except the timing cut. The four events shown in the bottom panel are single-scatter events passing the timing cut but are significantly outside the $2 \sigma$ nuclear recoil band. The event with very low ionization yield is likely an alpha-induced nuclear recoil. This event class is not expected to produce background in the signal region. Two events are in detectors with an exterior surface and contribute to our leakage estimate. The last event occurs in a detector with two internal surfaces, and $2.1_{-0.6}^{+0.8}(\mathrm{stat})_{-0.2}^{+0.3}$ (syst) events of this type are expected.

Figure 3 (upper panel) shows the Poisson 90\% C.L. upper limit on the spin-independent WIMP-nucleon (SI) cross section derived from this data (upper solid curve), based on standard assumptions about the galactic halo [7]. The minimum lies at $6.6 \times 10^{-44} \mathrm{~cm}^{2}$ for a $60 \mathrm{GeV} / c^{2}$ WIMP.

Our previous data from Soudan $[11,12]$ have been reanalyzed [18] yielding a slight improvement in sensitivity (upper curve) over our previous publications. A combined limit from all Soudan data (lower solid curve), using Yellin's Optimum Interval method [19] to account for observed events, gives an upper limit of $4.6 \times 10^{-44} \mathrm{~cm}^{2}$ at $90 \%$ C.L. for a WIMP mass of $60 \mathrm{GeV} / c^{2}$, a factor of $\sim 3$ stricter than our previously published limit. The combined CDMS SI limit (lower solid line) establishes the best sensitivity above $44 \mathrm{GeV} / c^{2}$, while XENON10 [20] (dashed) has greater sensitivity at low masses.

We also analyzed our data in terms of spin-dependent WIMP-neutron (SD) coupling and used the Ge form factor given in [21] to find a minimum upper limit of $2.7 \times$ $10^{-38} \mathrm{~cm}^{2}\left(1.8 \times 10^{-38} \mathrm{~cm}^{2}\right)$ at $90 \%$ C.L. for this data set (combined Soudan data). Figure 3 (lower panel) shows this limit and selected previous results. The combined SD CDMS limit verifies much of the cross section range covered by XENON10 [20].

CDMS has maintained high dark matter discovery potential by limiting expected backgrounds to less than one event in the signal region. The CDMS II results set the best WIMP sensitivity for spin-independent WIMP-nucleon interactions over a wide range of WIMP masses. Our
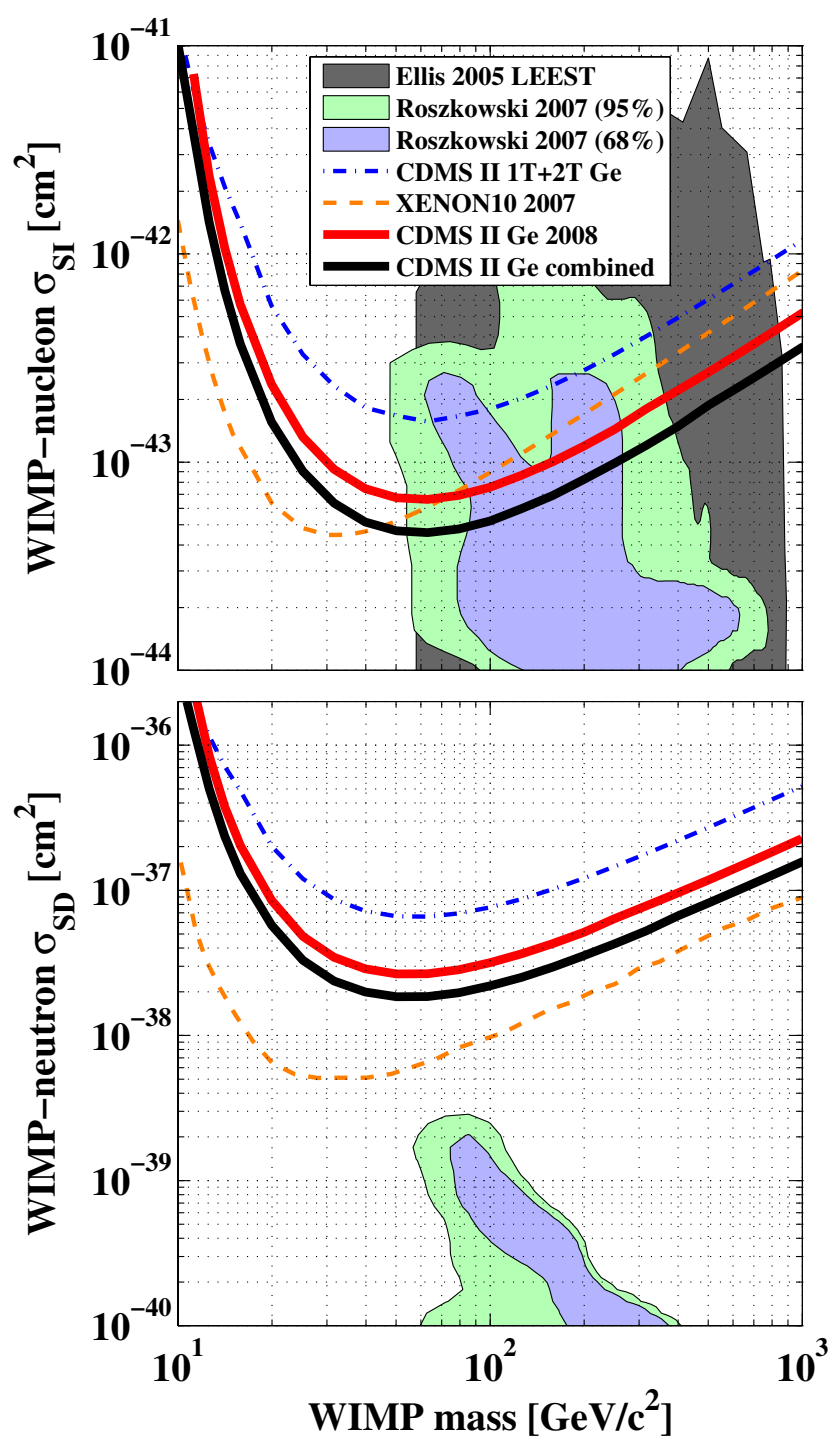

FIG. 3 (color online). Spin-independent WIMP-nucleon (SI) and spin-dependent WIMP-neutron (SD) cross-section upper limits (90\% C.L.) versus WIMP mass. In each panel, the curves represent $90 \%$ C.L. upper limits on the scattering cross section from this work and other recent experiments. Shaded regions represent parameter ranges expected from supersymmetric models described in [22,23]. Data courtesy of [24].

new limits cut significantly into previously unexplored regions of the central parameter space predicted by supersymmetry.

The CDMS collaboration gratefully acknowledges Patrizia Meunier, Daniel Callahan, Pat Castle, Dave Hale, Susanne Kyre, Bruce Lambin, and Wayne Johnson for their contributions. This work is supported in part by the National Science Foundation (Grant Nos. AST9978911, PHY-0542066, PHY-0503729, PHY-0503629, PHY-0503641, PHY-0504224, and PHY-0705052), by the Department of Energy (Contracts DE-AC03-76SF00098, DE-FG02-91ER40688, DE-FG03-90ER40569, and DEFG03-91ER40618), by the Swiss National Foundation 
(SNF Grant No. 20-118119), and by NSERC Canada (Grant SAPIN No. 341314-07).

[1] D. N. Spergel et al. (WMAP Collaboration), Astrophys. J. Suppl. Ser. 170, 377 (2007); M. Tegmark et al. (SDSS Collab.), Phys. Rev. D 69, 103501 (2004).

[2] G. Steigman and M.S. Turner, Nucl. Phys. B 253, 375 (1985).

[3] B. W. Lee and S. Weinberg, Phys. Rev. Lett. 39, 165 (1977); S. Weinberg, ibid. 48, 1303 (1982).

[4] G. Jungman, M. Kamionkowski, and K. Griest, Phys. Rep. 267, 195 (1996); G. Bertone, D. Hooper, and J. Silk, ibid. 405, 279 (2005).

[5] M. W. Goodman and E. Witten, Phys. Rev. D 31, 3059 (1985).

[6] R. J. Gaitskell, Annu. Rev. Nucl. Part. Sci. 54, 315 (2004).

[7] J. D. Lewin and P. F. Smith, Astropart. Phys. 6, 87 (1996).

[8] R. Bernabei et al. (DAMA/LIBRA Collaboration), Eur. Phys. J. C 56, 333 (2008); Nucl. Instrum. Methods Phys. Res., Sect. A 592, 297 (2008).

[9] E. A. Baltz, M. Battaglia, M. E. Peskin, and T. Wizansky, Phys. Rev. D 74, 103521 (2006).

[10] K. D. Irwin et al., Rev. Sci. Instrum. 66, 5322 (1995); T. Saab et al., AIP Conf. No. 605 (AIP, New York, Proc. 2002), p. 497.
[11] D. S. Akerib et al. (CDMS Collab.), Phys. Rev. D 72, 052009 (2005).

[12] D. S. Akerib et al. (CDMS Collab.), Phys. Rev. Lett. 96, 011302 (2006); D. S. Akerib et al. (CDMS Collab.), Phys. Rev. D 73, 011102 (2006).

[13] CDMS Collab. (to be published).

[14] A. Fassò et al., Report Nos. CERN-2005-10, INFN/ TC_05/11, SLAC-R-773, CERN, INFN, SLAC, 2005; A. Fassò et al., arXiv:hep-ph/0306267.

[15] J. S. Hendricks et al., Report No. LA-UR-08-1808; available from http://mcnpx.lanl.gov/LA-UR-08-1808.pdf.

[16] J. Allison et al., IEEE Trans. Nucl. Sci. 53, 270 (2006); S. Agostinelli et al., Nucl. Instrum. Methods Phys. Res., Sect. A 506, 250 (2003).

[17] D.S. Leonard et al. (EXO Collab.), Nucl. Instrum. Methods Phys. Res., Sect. A 591, 490 (2008).

[18] R. W. Ogburn, Ph.D. thesis, Stanford University, 2008; FERMILAB Report No. FERMILAB-THESIS-2008-33.

[19] S. Yellin, Phys. Rev. D 66, 032005 (2002).

[20] J. Angle et al. (XENON Collab.), Phys. Rev. Lett. 100, 021303 (2008).

[21] J. Engel, Phys. Lett. B 264, 114 (1991).

[22] J. R. Ellis, K. A. Olive, Y. Santoso, and V. C. Spanos, Phys. Rev. D 71, 095007 (2005).

[23] L. Roszkowski et al., J. High Energy Phys. 07 (2007) 075.

[24] R. J. Gaitskell, V. Mandic, and J. Filippini, http://dmtools. brown.edu. 